Int. J. Dev. Biol. 55: 395-398

doi: $10.1387 / \mathrm{ijdb} .103217 \mathrm{fb}$

\title{
Anti-angiogenesis in cancer; met and unmet goals - an interview with Robert Kerbel
}

\author{
FRANCESCO BERTOLINI* \\ Laboratory of Hematology-Oncology, European Institute of Oncology, Milan, Italy
}

\begin{abstract}
Robert Kerbel is one of the most influential scientists in the field of cancer-related angiogenesis and anti-angiogenic therapies. Along with the late Judah Folkman, Dr. Kerbel has also pioneered the use of continuous low dose chemotherapy for cancer treatment, an approach which is now widely used for many types of cancer and is a promising backbone to be associated with novel targeted therapies. In this interview, Dr. Kerbel describes the steps that moved his career towards the angiogenesis field, the already achieved biological and clinical results and the unmet goals.
\end{abstract}

KEY WORDS: Vasculogenesis, metronomic chemotherapy, VEGF, anti-VEGF, TKI

Very rarely, if ever, l've found a well recognized leader in a given scientific field to be as friendly and willing to chat about science as Robert (Bob) Kerbel. Years ago, Bob was among the few already prominent cancer scientist with enough foresight to be ready to embrace and enlarge the vision of the late Judah Folkman about the role of cancer vessels as crucial players in cancer growth and metastases and as possible drug targets. Moreover, Bob has paralleled Dr. Folkman in the development of a new approach to the administration of chemotherapy, which has been thereafter defined as "metronomic", ie continuous, with no brakes and at low doses. This particular therapeutic approach, along with anti-angiogenic drugs, is now more and more used by clinical oncologists. It might seem enough for a scientific career, but I am sure that Bob has something novel in mind...

Your scientific career begun before the blooming of the "angiogenesis in cancer" concept. What were the steps that brought you to this field and what were the first aims of your research in angiogenesis?

The initial steps that brought me into the field are somewhat ironic in view of some of the current interests that I have in tumor angiogenesis and antiangiogenic therapy, and where some important aspects of the field are currently heading. In 1991, I published a paper in a journal called BioEssays in which I put forward a hypothesis that antiangiogenic drugs might represent a form of anti-cancer therapy that would be far less vulnerable to the phenomenon of acquired drug resistance. The basic premise revolved around the notion that the ultimate target of such drugs is not the genetically unstable, highly mutable cancer cell population per se-which because of their extensive mutability can acquire resistance, often rapidly, to virtually any anti-cancer drug treatment
- but rather a genetically stable host cell population, namely, the vascular endothelial cells comprising newly forming and sprouting neovasculature. In my mind, this presented the theoretical possibility that one might be able to use antiangiogenic drugs for prolonged periods without resistance developing to them. Of course we now know from clinical experience especially that this is not the case. It was interesting to note how often this paper was either cited or discussed in scientific meetings for the next ten years after it was published, as almost an accepted fact - but without any supporting evidence! The irony is that there is now literally a cottage industry of investigators studying many different and diverse mechanisms which appear responsible for the development of resistance, or resistance-like phenotypes - to antiangiogenic drugs such as bevacizumab, sorafenib, and sunitinib. My lab is now working actively in this area. So this is the irony. In fact when I realized the theory was incorrect I felt it was important that I should try and be amongst the first investigators to say so. And in fact, I believe I was the first. I had a paper published in Science in 2002 (Yu et al., Science 295: 1526-1528, 2002) in which we reported for the first time a model of acquired resistance to an antiangiogenic therapy, which in this case involved genetic mutations that led to selection of tumor cell subpopulations capable of surviving in highly hypoxic tumor environments created by the antiangiogenic drug therapy. Since then a number of other groups have published studies describing other mechanisms. The most popular of these is known as "evasive resistance" championed, in particular, by Douglas Hanahan. This term refers to the selection of variant subpopulations of tumor

Abbreviations used in this paper: bFGF, basic fibroblast growth factor; TKI, tyrosine kinase inhibitor; VEGF, vascular endothelial growth factor.

*Address correspondence to: Francesco Bertolini. European Institute of Oncology, via Ripamonti 435, 20141 Milan, Italy.
e-mail: francesco.bertolini@ieo.it - web: http://www.ieo.it/Italiano/AreaClinica/Pages/UnitłdiLaboratoriodiEmatoncologiaClinica.aspx

Final, author-corrected PDF published online: 27 Juy 2011.

ISSN: Online 1696-3547, Print 0214-6282

(C) 2011 UBC Press

Printed in Spain 
cells that can induce angiogenesis by alternate, compensatory, proangiogenic pathways driven by factors such as bFGF, IL-8, etc. after targeting the preeminent pathway of angiogenesis driven by VEGF and VEGF receptors.

Aside from studying the issue of resistance, the other major aim of my initial research in the field dealt with linking the field of oncogenes and tumor angiogenesis. Thus I had a paper published in 1995, in Cancer Research, which was the first report linking an oncogene, in this case ras activation/mutation, with VEGF upregulation and hence angiogenesis. That paper was not even accepted for review by Science-one of the bigger disappointments of my career, given the abundant literature that has since accumulated on oncogenes and angiogenesis. In this regard, we then went on to show in 1997 that EGF receptor and Her-2/erbB-2 activation/expression can also contribute to the angiogenic switch. Since then, there have been a very large number of papers describing the fact that almost every known oncogene or class of oncogene has the potential of contributing to angiogenesis, not just by upregulating VEGF, but by a plethora of other mechanisms, including downregulation of endogenous angiogenesis inhibitor such as thrombospondin-1, as well as upregulating a variety of proangiogenic effector molecules.

In spite of evidence of clinical activity, the exact mechanism of action of anti-angiogenic drugs is still a matter of controversy. What is your personal point of view?

I think the discussion and debate about the mode of action of bevacizumab is a compelling example of a molecularly targeted drug - in this case, the target is VEGF - about which we know so little about how it actually works - when it does work. First of all, as is well known bevacizumab when used to treat patients with advanced cancer in clinical trials involving unselected/unstratefied patient populations is known to have very little activity - despite showing evidence in many such patients of anti-vascular function such as reduction in blood flow and reduced microvessel density counts. In many ways, this does not make sense. Why does the drug as a monotherapy not work better? As a result, as is well known, it must be combined with something else - chemotherapy so far in successful phase III trials - to yield a discernable and significant clinical benefit. This has provoked a vigorous discussion (and debate) about how an antiangiogenic drug such as bevacizumab enhances chemotherapy. There are probably about a half a dozen theories that have been proposed and they are very different in nature! There is the vessel normalization hypothesis championed by Rakesh Jain suggesting that increased chemotherapy drug delivery can be caused by administration of such drugs, which is counterintuitive. Second, there is the notion that chemotherapy drugs may damage the neovasculature in tumors and thereby render them more sensitive to an anti-VEGF agent that normally would be cytostatic, but which acquires cytotoxic anti-endothelial cell effects, since VEGF is known to be a survival (protective) factor. Another theory, one which we have been actively working on, involves the idea that chemotherapy drugs can sometimes induce a rapid mobilization of various bone marrow derived cells, including endothelial progenitor cells which then can migrate to and colonize the drug-treated tumors and act to accelerate tumor repopulation. This adaptive, reactive host response can be blunted by certain antiangiogenic drug and in so doing increase the efficacy of the chemotherapy. This was work that we published in Science in 2006 and then in Cancer Cell in 2008 (Shaked et al., in both

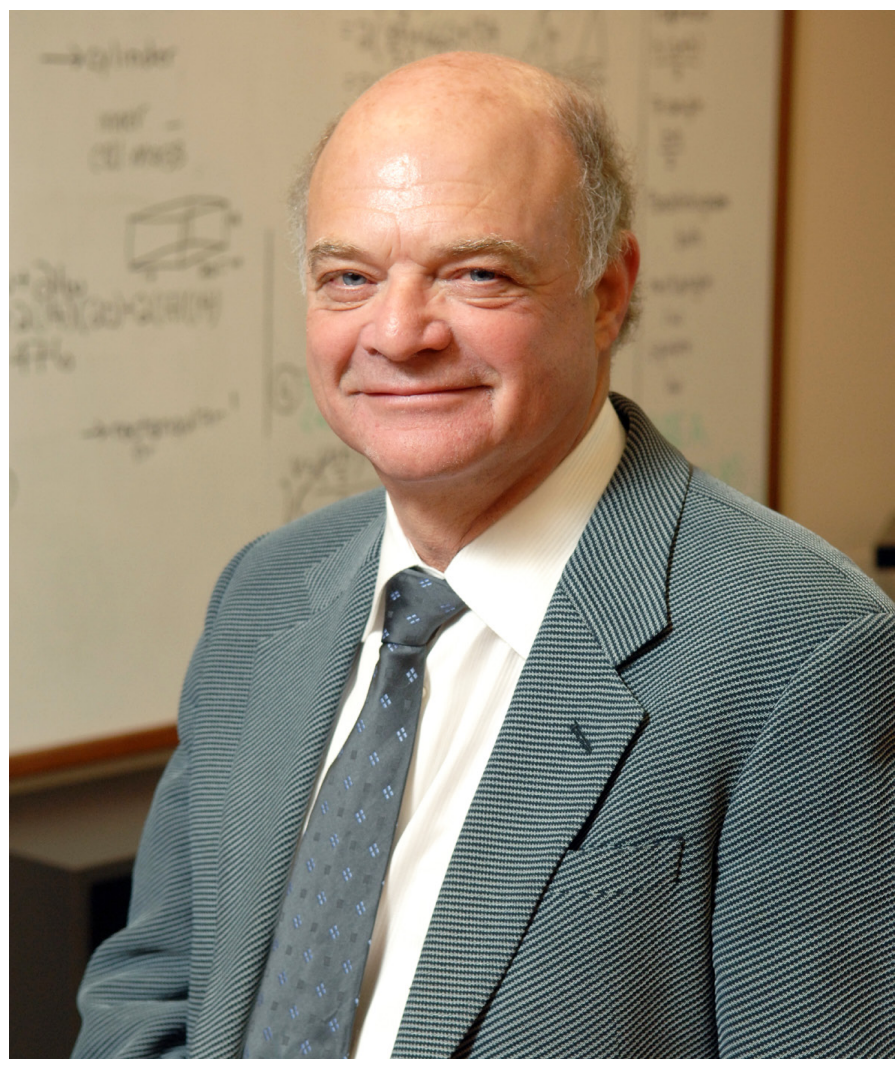

Fig. 1. Bob Kerbel, ready for a talk in 2011.

cases). By no means, does the story end there. For example, there are investigators who believe that in some cases VEGF is produced by tumor cells which also express VEGF receptors and as such, may act as an autocrine growth and survival factor. If so, in such cases blocking VEGF or VEGF receptors could have a direct impact on tumor cell, completely independent of inhibition of angiogenesis. And on it goes. I think it is unlikely that there will be a single dominant mechanism, and the importance of any particular one will depend on many different factors such as the nature of the chemotherapy and the antiangiogenic drug, and the nature and stage of the cancer being treated, and even the genetic background of the host. It's complex - but biologically fascinating.

At a given point, and before the clinical success of anti-angiogenic drugs, it became clear that cancer-related angiogenesis involves a variety of growth factors and related receptors and pathways. Were you surprised to see the clinical efficacy of molecules targeting only VEGF?

In some ways yes, and in some ways no. What I mean by that is the timing. I think that there was indeed a time when in addition to VEGF there were other proangiogenic growth factors that were thought to be extremely important, if not more so, in driving tumor angiogenesis, such as bFGF. However, by the late 1990s and at the beginning of the present decade there was accumulating evidence that VEGF was the preeminent growth factor driving angiogenesis in general, including tumor angiogenesis. In addition, there were the seminal 'knockout' studies published in the mid 1990s indicating that disruption of only a single VEGF allele in 
mice was sufficient to create an embryonic lethal phenotype. After those studies appeared, I really began to become convinced of the idea that targeting VEGF would eventually result in a discernible clinical benefit. So when the first phase III bevacizumab trial result was announced - the one with capecitabine in the $2^{\text {nd }}$ line metastatic breast cancer treatment setting - I was surprised and disappointed. But this disappointment was short lived when the next randomized bevacizumab/chemotherapy trial was announced about a year or so later, which led to the first approval of the drug, in this case in $1^{\text {st }}$ line colorectal cancer treatment with chemotherapy. I guess I also have to say that I'm not altogether surprised about the modest nature of the clinical benefits of the successful randomized phase III trials involving bevacizumab. Much has been made of these small benefits and they are generally viewed as a disappointment. However, such benefits are in line with almost every successful randomized phase III trial in medical oncology of any therapeutic in the advanced metastatic setting, where almost always the increased prolongations of progression-free or overall survival are measured in months. So it appears the same is true of antiangiogenic drugs. But it's also instructive to note that several antiangiogenic drugs have provided a benefit in treating the types of malignancy that historically have been notoriously difficult to systemic therapies such as hepatocellular carcinoma and renal cell carcinoma. One may add to this late stage non small cell lung cancer in the context of adding a biologic agent to a standard chemotherapy backbone such as carboplatin and paclitaxel: only bevacizumab has improved the outcome of chemotherapy while every other biologic agent has failed so far.

Do you believe some other pathways might have the same clinical relevance of VEGF for cancer therapy? And would you bet on drugs with multiple angiogenesis-related targets or on drugs with higher efficiency against a well defined target?

My bias, at least currently, is that for most types of malignancy, drugs which target a single pathway such as antibodies will be more effective, at least when combined with other therapies, particularly chemotherapy - including metronomic chemotherapy, as opposed to the multitargeting tyrosine kinase inhibitors. In this regard, I think one of the next most promising antibodies are those being developed to target angiopoietin-2 which binds to the tie- 2 receptor. There are also the peptibodies to ang-2 that may have promise. My bias is related to several factors, one being the general failure of the antiangiogenic TKIs to improve the efficacy of chemotherapy when used in such combination treatment along with the greater levels of toxicity that seem to be associated with most TKIs in comparison to antibodies. Nevertheless, there are also some intriguing possible breakthroughs looming for antiangiogenic TKIs, such as in metastatic thyroid cancer. Of course in such situations the activity of such drugs may be related to tyrosine kinases that are targeted which have nothing to do with inhibition of angiogenesis.

One of the visions of Dr. Folkman involved the prolonged use of anti-angiogenic drugs to prevent cancer cell escape from this therapy. Considering your studies about the possible pro-metastatic 'conditioning' effect of some TKIs in cancer-free mice before tumor cells are injected, is there a future for adjuvant or maintenance therapy with anti-angiogenic drugs?

I think there is a future for maintenance-type therapies using antiangiogenic drugs, but how robust it will be is certainly an open question. Currently I have some doubts about the impact of these drugs in the adjuvant setting - but this mainly concerns the use of TKIs - not antibodies. In addition, it is possible that the prometastatic effects my lab reported in recent preclinical investigations might apply primarily to situations involving relatively short term adjuvant therapy, and with a TKI. Normally adjuvant therapy is given for much longer periods, but given the toxicity of many of the TKIs it is a certainty that significant proportions of patients who are receiving adjuvant TKI therapy will decide to stop taking their treatment because of the various toxicities and the fact that they have early stage disease and may even be cured prior to taking this therapy. As is well known, the recently announced failed randomized adjuvant trial called 'CO8' involving bevacizumab plus chemotherapy, followed by bevacizumab maintenance therapy for 6 months in early stage II/III colorectal cancer did not meet its primary endpoint of a benefit in progression free survival at 3 years. However, there was a clearcut benefit in the interim analysis, undertaken at one year after initiation of treatment, which then progressively diminished over time. This suggests that Dr. Folkman was correct and that if the bevacizumab maintenance had been longer in duration, e.g. two years, the trial might have had a successful outcome. But of course this presents all sorts of other problems, even if true. One would then be faced with the dilemma with treating large number of patients, many of them already cured, with a very expensive drug that has side effects which occasionally can be serious in order to achieve what might be a relatively small percentage benefit in

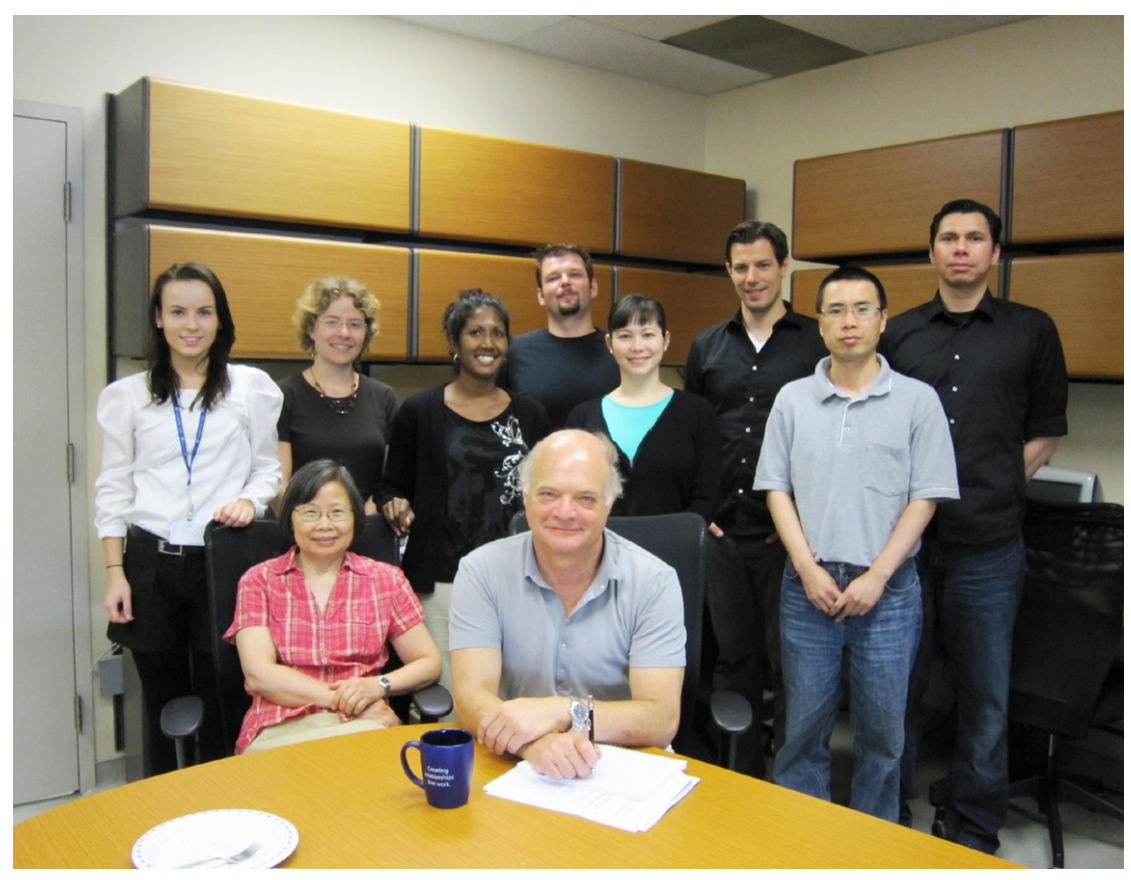

Fig. 2. Bob Kerbel and his lab's staff in 2011. 
cure in a large patient population. It's a real dilemma. Nevertheless, the results of $\mathrm{CO} 8$ do indeed suggest the possible benefit of longer term maintenance therapy with at least certain types of antiangiogenic drug, without resistance necessarily developing quickly. Hopefully, in the years ahead, safer and less expensive antiangiogenic drugs or treatments will be developed, making such chronic antiangiogenic therapy feasible and safe.

\section{What in your opinion is currently the most relevant unmet goal of the present generation of anti-angiogenic drugs?}

I would say the most relevant unmet goal is that they don't work better than is currently the case in the situations where they have shown a benefit, and that in other cases, they have not shown a benefit at all. So, for example, the lack of an overall survival benefit in metastatic breast cancer patients receiving an antiangiogenic based therapy represents, in a sense, an unmet medical need. And the failure thus far of antiangiogenic drugs to treat malignancies such as pancreatic cancer or early stage colorectal cancer also represents another example of an unmet medical need.

I am sure these unmet goals are shaping new scientific questions and many new studies in Bob's mind. Stay tuned for more breakthrough from his lab.

\section{Bob Kerbel's most significant recent contribution to the field of cancer angiogenesis}

RAK, J., MITSUHASHI, Y., BAYKO, L., FILMUS, J., SASAZUKI, T. and KERBEL, R.S. (1995) Mutant ras oncogenes upregulate VEGF/VPF expression: implications for induction and inhibition of tumor angiogenesis. Cancer Res., 55:4575-4580. A demonstration of the role of genetic instability in cancer-associated angiogenesis.

KLEMENT, G., BARUCHEL, S., RAK, J., MAN, S., CLARK, C., HICKLIN, D., BOHLEN, P. and KERBEL, R.S. (2000) Continuous low-dose therapy with vinblastine and VEGF receptor-2 antibody induces sustained tumor regression without overt toxicity. J. Clin. Invest., 105: R15-R24. The first report of the efficacy of the association of anti-angiogenesis and metronomic chemotherapy.

MUNOZ, R., MAN, S., SHAKED, Y., LEE, C.R., WONG, J., FRANCIA, G. and KERBEL, R.S. (2006) Highly efficacious non-toxic preclinical treatment for advanced metastatic breast cancer using combination oral UFT - cyclophosphamide metronomic chemotherapy. Cancer Res., 66: 3386-3391. The preclinical basis of metronomic chemotherapy for breast cancer.

SHAKED, Y., CIARROCCHI, A., FRANCO, M., LEE, C.R., MAN, S., CHEUNG, A.M., HICKLIN, D.J., CHAPLIN, D., FOSTER, F.S., BENEZRA, R. and KERBEL, R.S. (2006) Therapy-induced acute recruitment of circulating endothelial progenitor cells to tumors. Science, 313: 1785-1787. The demonstration of the contribution of endothelial progenitors to tumor resistance after therapy.

KERBEL, R.S. (2008) Tumor angiogenesis. New Engl. J. Med. 358: 2039-2049. A comprehensive review of the field with provocative hypotheses and foresight for future challenges.

EBOS, J.M.L., LEE, C.R., CRUZ-MUNOZ, W., BJARNASON, G.A., CHRISTENSEN, J.G. and KERBEL, R.S. (2009) Acceleration of metastasis after short-term treatment with a potent inhibitor of tumor angiogenesis. Cancer Cell, 15: 232-239. The first report of a possible risk for increased tumor invasiveness after the administration of tyrosine kinase inhibitors with anti-angiogenic activity. 


\section{Further Related Reading, published previously in the Int. J. Dev. Biol.}

See our recent Special Issue Placenta edited by Joan S. Hunt and Kent L. Thornburg at: http://www.ijdb.ehu.es/web/contents.php?vol=54\&issue=2-3

The seminal work of Werner Risau in the study of the development of the vascular system Domenico Ribatti

Int. J. Dev. Biol. (2010) 54: 567-572

The contribution of Roberto Montesano to the study of interactions between epithelial sheets and the surrounding extracellular matrix Domenico Ribatti

Int. J. Dev. Biol. (2010) 54: 1-6

Parallels in invasion and angiogenesis provide pivotal points for therapeutic intervention Suzanne A. Eccles

Int. J. Dev. Biol. (2004) 48: 583-598

Angiogenesis and apoptosis are cellular parameters of neoplastic progression in transgenic mouse models of tumorigenesis G Bergers, D Hanahan and L M Coussens

Int. J. Dev. Biol. (1998) 42: 995-1002

The chick embryo chorioallantoic membrane as a model for in vivo research on angiogenesis D Ribatti, A Vacca, L Roncali and F Dammacco Int. J. Dev. Biol. (1996) 40: 1189-1197

$$
5 \text { yr ISI Impact Factor }(2009)=3.253
$$



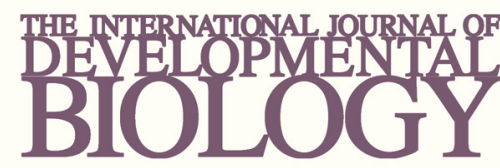

Volume 54 Nos. 6/7
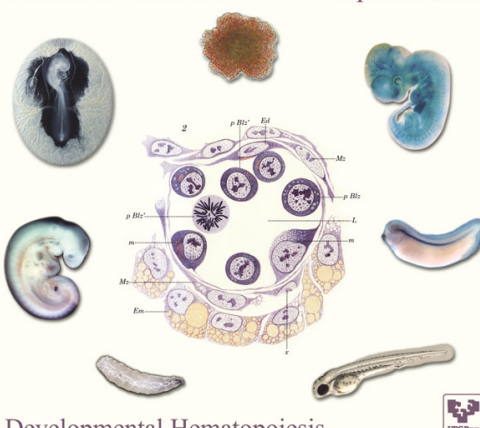

Developmental Hematopoiesis

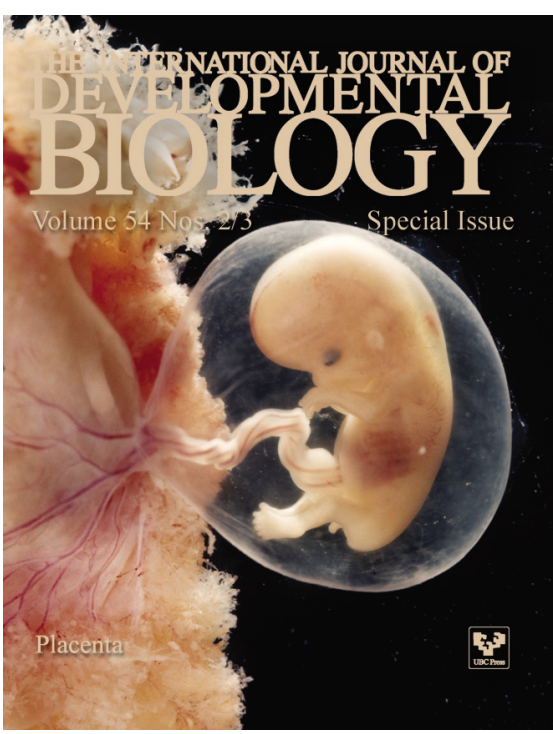

